\title{
The chemical signatures of the Large Magellanic Cloud globular clusters
}

\author{
Alessio Mucciarelli ${ }^{1}$ \\ ${ }^{1}$ Dipartimento di Astronomia, Università degli Studi di Bologna, Bologna, Italy \\ email: alessio.mucciarelli@studio.unibo.it
}

\begin{abstract}
We present the first results of a long-term project based on the analysis of highresolution optical spectra for a sample of Large Magellanic Cloud globular clusters. The final aim is to build a new, reliable metallicity scale for this cluster system and shed some light on the role played by the different chemical contributors (AGB, SN II and SN Ia), in order to understand the chemical enrichment history of the Large Magellanic Cloud. The analysis of 6 young and intermediate-age clusters and 11 field stars, observed with the UVES@FLAMES spectrograph, provides crucial information about the chemical composition of the dominant stellar population of the LMC. All these stars are metal-rich $([\mathrm{Fe} / \mathrm{H}] \sim-0.4$ dex $)$, with solarscaled $[\alpha / \mathrm{Fe}]$ ratios, that point toward an enrichment from SN Ia. Moreover, we observed a general depletion for the $[\mathrm{Al} / \mathrm{Fe}]$ ratio (indication of a chemical enrichment by metal-poor $\mathrm{SN}$ II) and a strong enhancement of the $[\mathrm{Ba} / \mathrm{Y}]$ ratio (likely due to the enrichment by metal-poor AGB stars).
\end{abstract}

Keywords. techniques: spectroscopic, stars: abundances, galaxies: evolution, galaxies: Individual (LMC), Magellanic Clouds, galaxies: star clusters

\section{The chemistry of the Large Magellanic Cloud: state of the art}

The Large Magellanic Cloud (LMC) is the nearest galaxy with a present-day starformation activity and represents a fundamental laboratory to study the stellar populations. One of its most important peculiarities is the wide age and metallicity distribution covered by its stellar clusters. Several age families for these objects have been recognized: an old population, with clusters coeval with the Galactic ones, an intermediate-age population, including clusters with ages between $\sim 1$ and $\sim 3$ Gyr, and finally numerous clusters younger than $\sim 1$ Gyr. A lot of information about the metallicity of the stellar content of the LMC derived by Ca II triplet surveys was presented by several authors in the last two decades, e.g., Olszewski et al. (1991), Cole et al. (2005), Grocholski et al. (2006), and Carrera et al. (2007).

One of the most severe limitations in our knowledge of the LMC stellar populations is the lack of homogeneous age and metallicity scales, fundamental tools to derive a reliable age-metallicity relation and well understand the chemical evolution of this environment. Few works based on high-resolution spectra are actually available for the LMC stellar content, and our knowledge of the chemical signatures of these stellar populations is yet partial. Hill et al. (2000) discussed the first measurements from high-resolution spectroscopy for some LMC clusters, showing the abundance patterns for $\mathrm{Fe}, \mathrm{O}$ and $\mathrm{Al}$. A detailed screening of the chemistry in 4 old LMC clusters has been presented by Johnson et al. (2006). Recently, Pompéia et al. (2008) find some peculiarities in the chemical composition of LMC field stars, such as the systematic depletion of $\mathrm{Ni}$ and iron-peak elements and peculiar and complex abundance pattern of the neutron-capture elements. 


\section{The global project: towards an age-metallicity relation}

We present the first results of a long-term spectro-photometric project devoted to obtain accurate and reliable metallicity and age estimates for a sample of template clusters. The whole spectroscopic database obtained by using the ESO facility FLAMES@VLT includes 9 stellar clusters, spanning the entire age range covered by the LMC cluster system. We have selected 2 young clusters with ages $\lesssim 1$ Gyr (namely NGC 2157 and 2108, Mucciarelli et al. (2009)), 4 intermediate-age clusters (NGC 1651, 1783, 1978 and 2173, Ferraro et al. (2006) and Mucciarelli et al. (2008)) and 3 old clusters (NGC 1786, 2210 and 2257, in preparation). Moreover, 11 giant stars turned out not to be cluster members but to belong to the LMC field and we have analysed also these stars in order to compare the abundance patterns inferred from cluster and field stars.

In the following we summarize the principal aims of this project:

- The definition of a new, homogeneous metallicity scale for a representative sample of LMC template clusters, based on the latest generation of high-resolution spectrographs.

- The detailed study of the abundance patterns for the main elemental groups, as iron-peak, light Z-odd, $\alpha$ and neutron-captures elements, in order to well describe the chemical enrichment history of the stellar populations in the LMC.

- The combination of the information about the overall metallicity, $[\mathrm{M} / \mathrm{H}]$, of each target cluster with the high-resolution, photometric database available in the HST archive will provide accurate estimates of the age for these objects. The first results of this procedure have been discussed in Mucciarelli et al. (2007a,b). In Figs. 1 and 2 we report the Color-Magnitude Diagram of the two intermediate-age clusters NGC 1978 and 1783, obtained with ACS@HST photometry. For these two clusters we have derived an age of 1.9 and 1.4 Gyr, respectively.

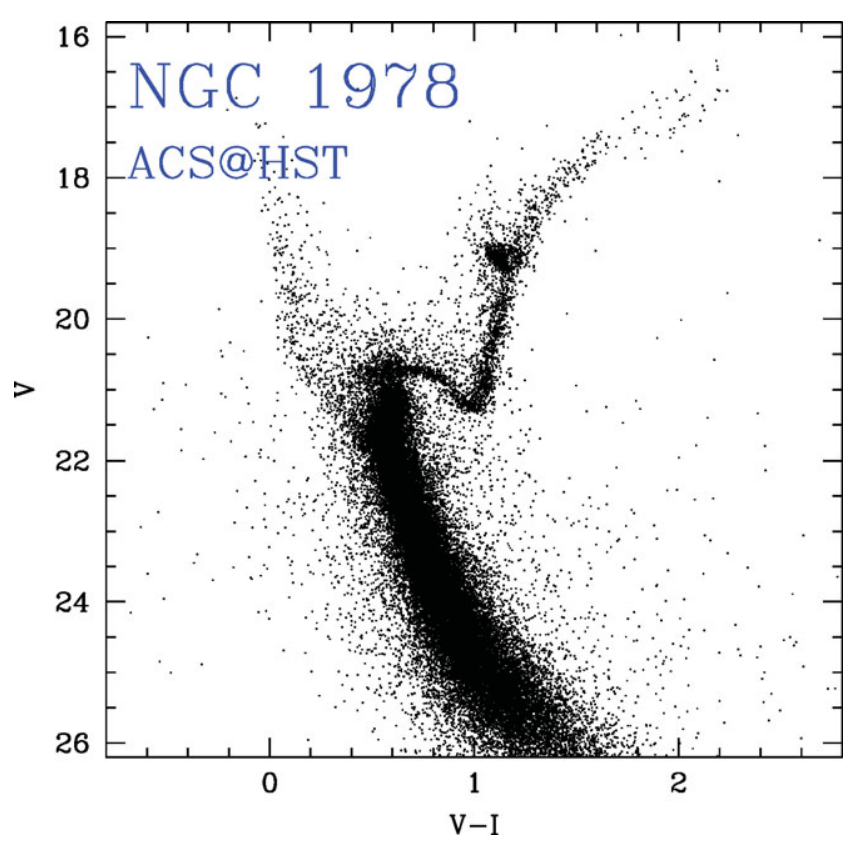

Figure 1. The color-magnitude diagram for the LMC cluster NGC 1978, obtained with ACS@HST. 


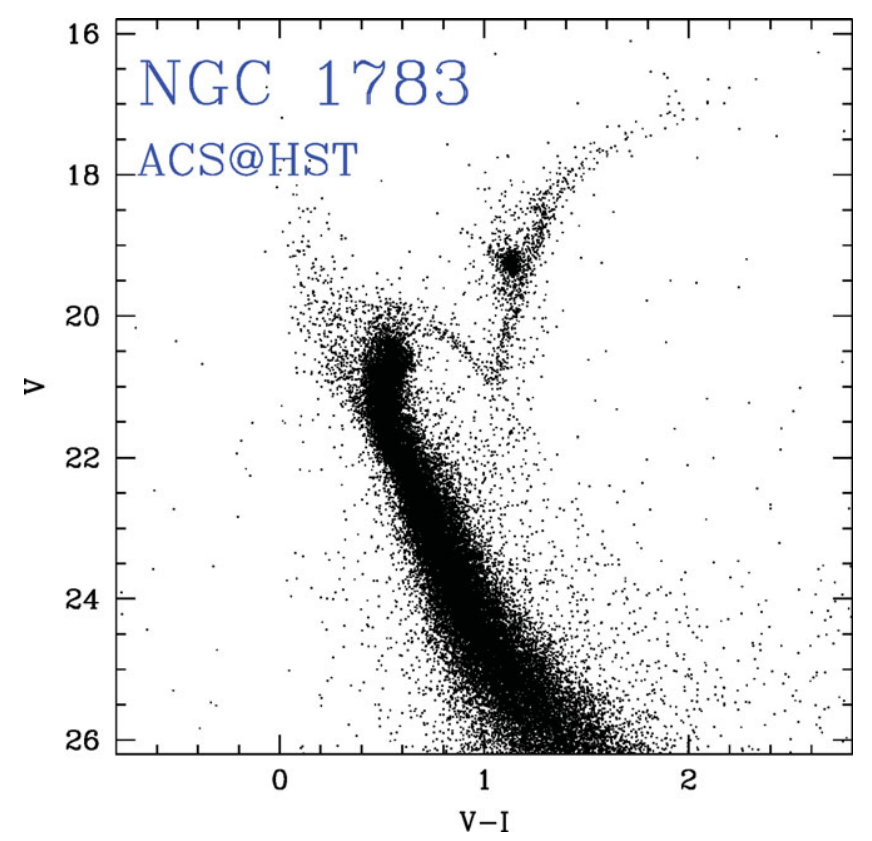

Figure 2. The color-magnitude diagram for the LMC cluster NGC 1783, obtained with ACS@HST.

\section{The chemical composition}

All 6 LMC clusters and 11 field stars analysed here turn out to be metal-rich, with an average value for the clusters of $[\mathrm{Fe} / \mathrm{H}]=-0.35 \operatorname{dex}(\sigma=0.11 \mathrm{dex})$, while the field stars exhibit an average iron content of $[\mathrm{Fe} / \mathrm{H}]=-0.49 \mathrm{dex}(\sigma=0.10 \mathrm{dex})$. This finding appears to be consistent with the metallicity distribution for the LMC field inferred by different works, in particular the recent studies based on the Ca II triplet by Cole et al. (2005) $([\mathrm{Fe} / \mathrm{H}]=-0.37 \pm 0.15 \mathrm{dex})$, Grocholski et al. $(2006)([\mathrm{Fe} / \mathrm{H}]=-0.48 \pm 0.09 \mathrm{dex})$ and Carrera et al. $(2007)([\mathrm{Fe} / \mathrm{H}] \sim-0.5 \mathrm{dex})$.

\section{1. $\alpha$-elements}

The $\alpha$-elements are produced mainly by massive stars (and ejected via the SN II events), with a smaller contribution by SN Ia, while the iron is produced mainly by SN Ia. For this reason, the $[\alpha / \mathrm{Fe}]$ ratio turns out to be a powerful diagnostic of the relative contribution of SN II to SN Ia. Our stars show sub-solar ratios for $[\mathrm{O} / \mathrm{Fe}]$ and $[\mathrm{Ca} / \mathrm{Fe}]$, while $[\mathrm{Mg} / \mathrm{Fe}]$, $[\mathrm{Si} / \mathrm{Fe}]$ and $[\mathrm{Ti} / \mathrm{Fe}]$ match the Galactic patterns. Fig. 3 reports the average value of $[\mathrm{Mg} / \mathrm{Fe}],[\mathrm{Ca} / \mathrm{Fe}]$ and $[\mathrm{Ti} / \mathrm{Fe}]$ of our stars (the red circles are the average values for the LMC clusters and the red asterisks the individual LMC field stars) in comparison with other high-resolution spectroscopic databases: grey points indicate the Galactic stars (Venn et al. 2004; Reddy et al. 2006) and blue points the Sgr dSph giant stars (Sbordone et al. 2007). The LMC stars seem to fall between the envelope described by the Galactic stars and the one from the Sgr dSph. The solar $[\alpha / \mathrm{Fe}]$ ratios observed in these LMC stars indicates an enrichment from SN Ia.

\section{2. $\mathrm{Na}$ and $\mathrm{Al}$}

There are two main channels in order to produce $\mathrm{Na}$ and $\mathrm{Al}$. The first one is linked to the SN II, in order to provide the extra neutron present in the dominant isotope of both 


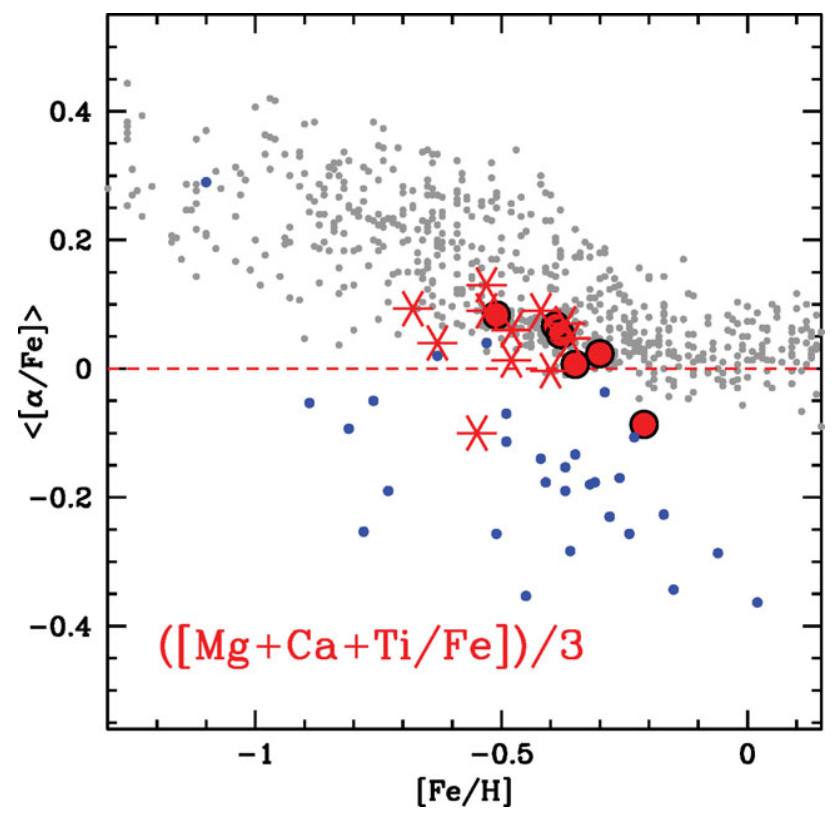

Figure 3. The behaviour of $[\alpha / \mathrm{Fe}]$ ratio (defined as the average between $[\mathrm{Mg} / \mathrm{Fe}],[\mathrm{Ca} / \mathrm{Fe}]$ and $[\mathrm{Ti} / \mathrm{Fe}])$ as a function of $[\mathrm{Fe} / \mathrm{H}]$. Red points are the average values for the target LMC clusters, red asterisks are the LMC field stars, grey points indicate the Galactic stars by Venn et al. (2004) and Reddy et al. (2006) and the blue points are the giant stars of the Sgr dSph by Sbordone et al. (2007).

element $\left({ }^{23} \mathrm{Na}\right.$ and $\left.{ }^{27} \mathrm{Al}\right)$. The $\mathrm{Na}$ and $\mathrm{Al}$ production is controlled by the neutron excess and in this way the yields for these elements becomes metallicity-dependent. Another possible channel to produce $\mathrm{Na}$ and $\mathrm{Al}$ is linked to the AGB stars, in which proton capture reactions can occur through the $\mathrm{NeNa}$ and $\mathrm{MgAl}$ cycles. This mechanism is invoked in order to explain the so-called anticorrelations in the observed Galactic globular clusters. In our cluster sample we find a very high degree of homogeneity for $\mathrm{Na}, \mathrm{Al}, \mathrm{O}$ and $\mathrm{Mg}$, without hints of possible intrinsic chemical anomalies. For this reason, we can consider the AGB channel not very efficient and the $\mathrm{Na}$ and Al production attributed to the SN II events. Our target stars, both cluster and field stars, exhibit a general depletion of these two abundance ratios (see Fig. 4), with a strong depletion in the $[\mathrm{Al} / \mathrm{Fe}]$ ratio $(\sim-0.40 \mathrm{dex})$. This finding seems to indicate that the interstellar medium from which these stars were born had been enriched with the ejecta of low-metallicity SN II.

\subsection{Neutron-capture elements}

The LMC stars show a peculiar and dichotomic behaviour for the s-process elements. The elements belonging to the first peak (such as $\mathrm{Y}$ and $\mathrm{Zr}$ ) show a general depletion with respect to the solar value $(\sim-0.4 \mathrm{dex})$, while the elements of the second peak (such as $\mathrm{Ba}$, La and $\mathrm{Nd})$ turn out to be enhanced $(\sim+0.5 \mathrm{dex})$. The $[\mathrm{Ba} / \mathrm{Y}]$ ratio (indicated by Venn et al. (2004) as an important diagnostic to study this family of elements) turns out to be strongly enhanced, with values of $[\mathrm{Ba} / \mathrm{Y}] \sim+0.80$ dex, similar to the observed pattern in Sgr dSph but in evident disagreement with the Galactic trend, that exhibits a solar-scaled pattern at the same metallicity level. The interpretation of these abundance patterns is complicated by the multiplicity of the nucleosynthetic sites. 


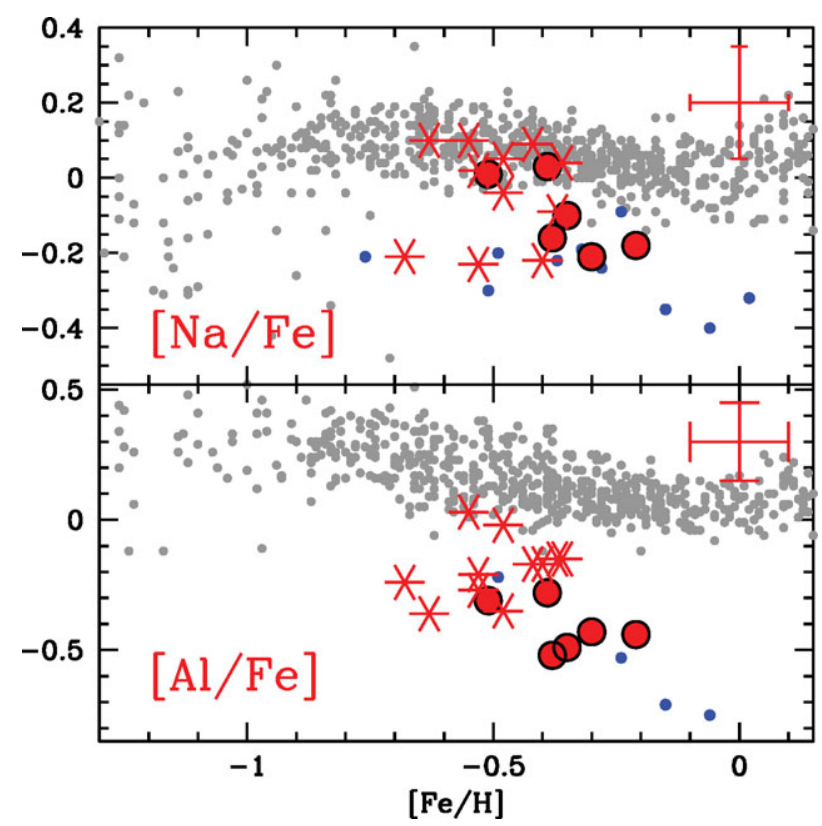

Figure 4. The behaviour of $[\mathrm{Na} / \mathrm{Fe}]$ (upper panel) and $[\mathrm{Al} / \mathrm{Fe}]$ (lower panel) ratios as a function of $[\mathrm{Fe} / \mathrm{H}]$. Same symbols of Fig. 3 .

Theoretical predictions by Travaglio et al. (2004) indicate that the AGB yields for this kind of elements are metallicity-dependent. A high value of the $[\mathrm{Ba} / \mathrm{Y}]$ ratio could suggest a major pollution of the gas by low-metallicity AGB stars.

\section{Conclusions}

The analysis of high-resolution spectra of 6 young-intermediate-age clusters and 11 field stars of the LMC has revealed some chemical signatures of the dominant stellar populations of this galaxy. (1) All these stars, both of field and cluster, are metal-rich, with an iron content that confirms the previous analysis based on the Ca II triplet; (2) the LMC stars exhibit solar $[\alpha / \mathrm{Fe}]$ ratios, indication of the chemical contribution from the ejecta of SN Ia; (3) all the target stars show low values of $[\mathrm{Na} / \mathrm{Fe}]$ and $[\mathrm{Al} / \mathrm{Fe}]$ ratios when compared with the Galactic Disk stars; (4) the $[\mathrm{Ba} / \mathrm{Y}]$ ratio turns out to be enhanced $(+0.5 /+1.0 \mathrm{dex})$, pointing toward an enrichment by metal-poor AGB stars.

\section{References}

Carrera, R., Gallart, C., Hardy, E., Aparicio, A., \& Zinn, R. 2008, AJ, 135, 836

Cole, A. A., Tolstoy, E., Gallagher III, J. S., \& Smecker-Hane, T.A. 2005, AJ, 129, 1465

Ferraro, F. R., Mucciarelli, A., Carretta, E., \& Origlia, L. 2006, ApJ, 133, L3

Grocholski, A. J., Cole, A. A., Sarajedini, A., Geisler, D., \& Smith, V. V. 2006, AJ, 132, 1630

Hill, V., François, P., Spite, M., Primas, F., \& Spite, F. 2000, A $\& A S, 364,19$

Johnson, J. A., Ivans, I., \& Stetson, P. 2006, ApJ, 640, 801

Mucciarelli, A., Ferraro, F. R., Origlia, L., \& Fusi Pecci, F. 2007a, AJ, 133, 2053

Mucciarelli, A., Origlia, L., \& Ferraro, F. R. 2007b, AJ, 134, 1813

Mucciarelli, A., Carretta, E., Origlia, L., \& Ferraro, F. R. 2008, AJ, 136, 375

Mucciarelli, A., Origlia, L., \& Ferraro, F. R. 2009, AJ, submitted 
Olszewski, E. W., Schommer, R. A., Suntzeff, N. B., \& Harris, H. C. 1991, AJ, 101, 515

Pompéia, L., Hill, V., Spite, M., et al. 2008, A\& A, 480, 379

Reddy, B. E., Lambert, D. L., \& Allende Prieto, C. 2006, MNRAS, 367, 1329

Sbordone, L., Bonifacio, P., Buonanno, R., Marconi, G., Monaco, L., \& Zaggia, S. 2007, AשEA, 465,815

Travaglio, C., Gallino, R., Arnone, E., Cowan, J., Jordan, F., \& Sneden, C. 2006, ApJ, 601, 864

Venn, K. A., Irwin, M. Shetrone, M. D., Tout, C. A., Hill, V., \& Tolstoy, E. 2004, AJ, 128, 1177

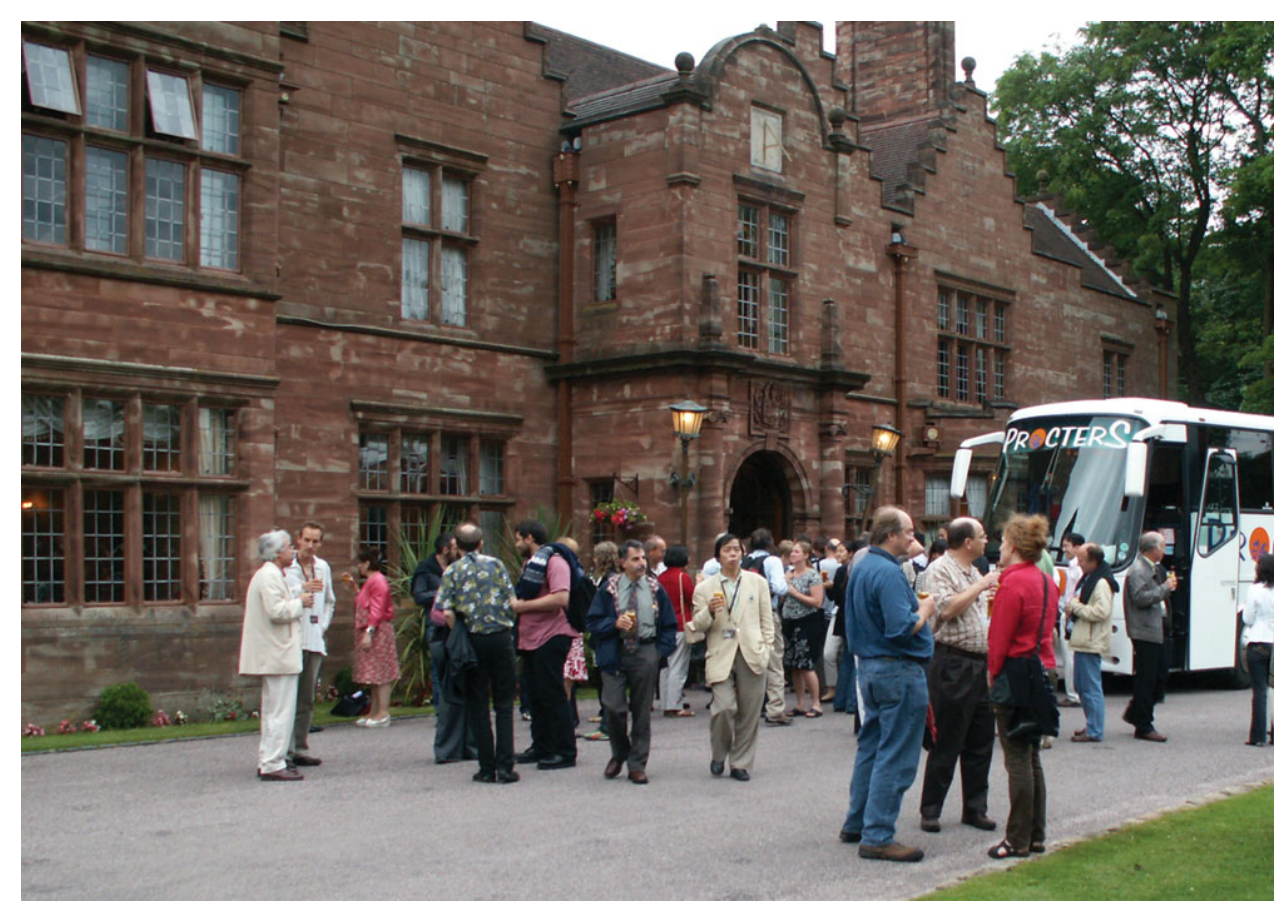

Arrival at Wrenbury Hall, after a leisurely ride through the Cheshire countryside. 